\title{
Study of nitrate contaminants removal from groundwater on copper modified BDD electrode
}

\author{
Peijing Kuang ${ }^{1,2,4}$, Yubo Cui ${ }^{1,2}$, Chuanping Feng ${ }^{3}$, and Yasuaki Einaga ${ }^{4,5^{*}}$ \\ ${ }^{1}$ Key Laboratory of Biotechnology and Bioresources Utilization, Ministry of Education, Dalian Minzu University, 18 Liaohe Road West, \\ Dalian Economic and Technological Development Zone, Dalian 116600, China \\ ${ }^{2}$ College of Environment and Resources, Dalian Minzu University, Dalian, 116600, China \\ ${ }^{3}$ School of Water Resources and Environment, China University of Geosciences (Beijing), 29 Xue Yuan Road, Haidian District, Beijing \\ 100083, China \\ ${ }^{4}$ Department of Chemistry, Keio University, 3-14-1 Hiyoshi, Yokohama 223-8522, Japan \\ ${ }^{5}$ JST-ACCEL, 3-14-1 Hiyoshi, Yokohama 223-8522, Japan
}

\begin{abstract}
The electrochemical nitrate reduction by using boron-doped diamond (BDD) and copper modified boron-doped diamond (Cu-BDD) electrodes was investigated at various potentials. Nitrate reduction efficiency and the products distribution was strongly dependent on the applied potential for both electrodes. The highest nitrate reduction efficiency of $77 \%$ was obtained at $-2.0 \mathrm{~V}$ ( $\mathrm{vs}$. $\mathrm{Ag} / \mathrm{AgCl})$ by using $\mathrm{Cu}$-BDD. Compared with BDD electrode, nitrate reduction on $\mathrm{Cu}$-BDD electrode occurred at more positive potential. Copper oxides formed on BDD surface efficiently promoted enhanced conductivity of electrode to promote electrons transfer during nitrate reduction process. Meanwhile, the catalytic ability of copper was also conductive to the nitrate transformation. Therefore, the developed $\mathrm{Cu}$-BDD would be a promising approach for efficient nitrate removal from groundwater.
\end{abstract}

\section{Introduction}

Nitrate concentration has increased in groundwater and soil because of the heavy use of nitrogen based fertilisers and the discharge of agricultural effluent, causing the serious nitrate pollution [1]. The daily human intake of nitrate is predominantly caused by food and drinking water, and the exposure of nitrate would result in "blue baby syndrome" in infants [2], liver damage [3] and cancer [4]. Therefore the maximum permissible level of nitrate is set at by $50 \mathrm{mg} / \mathrm{L}$ (ca. $0.8 \mathrm{mM}$ ) European drinking water directive. Based on this urgent situation, considerable researches have been made towards nitrate removal, such as biological denitrification, adsorption, chemical catalysis and electrochemistry.

As one of clean technology, electroreduction was widely used to abate nitrate from the polluted groundwater since it offers high efficiency, convenience and low cost operation. However, the complex nitrate electroreduction process depends strongly on the experimental parameters such as the $\mathrm{pH}$, electrolyte and the electrode material, which results in a mass of nitrite, ammonia and some other undesirable by-products. In this sense, a mass of materials have been explored to promote nitrate transformation towards nitrogen gas.

Born doped diamond (BDD), which has been known as its super stability, ruggedness, low background current and wide potential window, has been widely studied for electrochemical nitrate reduction. BDD was proved to have a high selectivity of nitrogen gas formation. Kuang et al found that BDD electrode had the higher nitrogen gas selectivity of $45.2 \%$ than $\mathrm{Cu}$ of $8 \%$ for nitrate reduction under the same electrolytic conditions, which might result from the inhibition of ammonia generation by the wide potential of BDD [5]. However, the high overpotential was still needed to reduce nitrate, and the semi-conductive $\mathrm{BDD}$ was to the disadvantage of electrons transfer, which retarded electrochemical nitrate reduction.

An alternative method was proved to be the construction of modified BDD using catalytic materials, which has shown the electro-catalytic characterization for the electro-reduction of nitrate. In particular, copper was widely used to modify BDD due to its low-cost and easy to get. Researchers found that copper modified BDD (Cu-BDD) had the high sensitivity and low limits for detecting nitrate [6]. Cu-BDD also had the higher kinetics for the nitrate reduction process owing to the excellent catalytic capacity of copper [7]. Some researchers also found that $\mathrm{Cu}-\mathrm{BDD}$ with the higher conductivity had the lower potential for nitrate reduction into ammonia. Neverless, nitrate reduction efficiency and their products distribution are still unknown. On the other hand, nitrate reduction was proved to strongly depend on applied potential, which has not been explored using $\mathrm{Cu}-\mathrm{BDD}$. In our study, we examined $\mathrm{Cu}-$ $\mathrm{BDD}$ for the electrochemical reduction of nitrate and the selectivity of nitrogen gas under different potentials.

* Corresponding author: einaga@chem.keio.ac.jp 


\section{Experimental}

\subsection{Reagents and materials}

Regents for solution preparation were purchased from Wako pure chemical industries and used without further purification. Detection reagents for nitrate, nitrite, and ammonium were purchased from Merck KGaA. prepared using Ultrapure water used for aqueous solutions was obtain from a Simply-Lab water system (DIRECT-Q 3 UV, Millipore) with the resistivity of 18 $\mathrm{M} \Omega \mathrm{cm}$.

\subsection{Preparation of the working electrodes}

BDD electrode was grown on the $\mathrm{Si}(111)$ wafer by microwave plasma-assisted chemical vapor deposition (MPCVD) system (AX6500, CORNES Technologies Corp.), and the preparation process was same as our previous report [8]. Copper particles were electrodeposited on the BDD surface by chronoamperometry method at the potential of $-0.6 \mathrm{~V}$, using $15 \mathrm{ml}$ of $1 \mathrm{mM} \mathrm{CuSO}_{4} / 0.1 \quad \mathrm{M} \mathrm{H}_{2} \mathrm{SO}_{4}$. The modification process lasted for $300 \mathrm{~s}$ to explored the electrode performance of electrochemical nitrate reduction. $\mathrm{Cu}-\mathrm{BDD}$ was recovered by immersing electrode in aqua regia for 10 minutes and ultrasonically cleaning in ultra-pure water for $5 \mathrm{~min}$.

\subsection{Electrochemical measurements}

Electrochemical nitrate reduction experiments were carried out in a two-compartment cell separated by a Nafion membrane, in which a typical three-electrode system was constructed using $\mathrm{Cu}$-BDD (working electrode), $\mathrm{Pt}$ (counter electrode) and $\mathrm{Ag} / \mathrm{AgCl}$ (saturated $\mathrm{KCl}$ ) (reference electrode) [9].Catholyte (50 mL $1.0 \mathrm{~g} \mathrm{~L}^{-1} \mathrm{Na}_{2} \mathrm{SO}_{4}$ containing $50 \mathrm{mg}-\mathrm{N} \mathrm{L}^{-1} \mathrm{NaNO}_{3}$ ) and anolyte $\left(50 \mathrm{~mL} 1.0 \mathrm{~g} \mathrm{~L}^{-1} \mathrm{Na}_{2} \mathrm{SO}_{4}\right)$ were circulated from storage bottles into chambers by a peristaltic pump (Masterflex, Cole Parmer) at flow rate of $100 \mathrm{~mL} \mathrm{~min}^{-1}$. The performance of $\mathrm{Cu}$-BDD for electrochemical nitrate reduction was explored using chronoamperometry at different potential using a potentiostat (Autolab PGSTAT204, Metrohm Autolab B.V.), during which the nitrate reduction efficiency and its products distribution were obtained after $2 \mathrm{~h}$ electrolysis.

\subsection{Preparation of the working electrodes}

The liquid products (nitrate, nitrite, and ammonium ions) were analyzed by a visible spectrophotometer (Photolab 6100VIS, WTW GmbH), based on the colorimetric method previously described [5]. Gas products (nitrogen and hydrogen gas) generated during electrolysis were measured using by gas chromatograph with a thermal conductivity detector (GC-2014, Shimadzu Corp.).

\section{Results and discussion}

\subsection{Characterization}

Electrodes were characterized by Scanning Electron Microscope (SEM) and Energy Dispersive X-Ray Fluoresence Spectrometer (EDX) to examine the surface morphology and deposited copper content of the BDD electrodes, before and after electrodeposition. Before deposition, SEM images of BDD showed the welldefined, crystal facets (Fig. 1a). After electrodeposition, large amount of particles could be observed on BDD surface with the average diameter of $101 \mathrm{~nm}$ (Fig. 1b and 1c). After the recovery of Cu-BDD, BDD electrode was also examined by SEM. Results showed that the surface morphology of BDD kept the same look of unused one, proving the high stability of BDD as previous study reported [13].

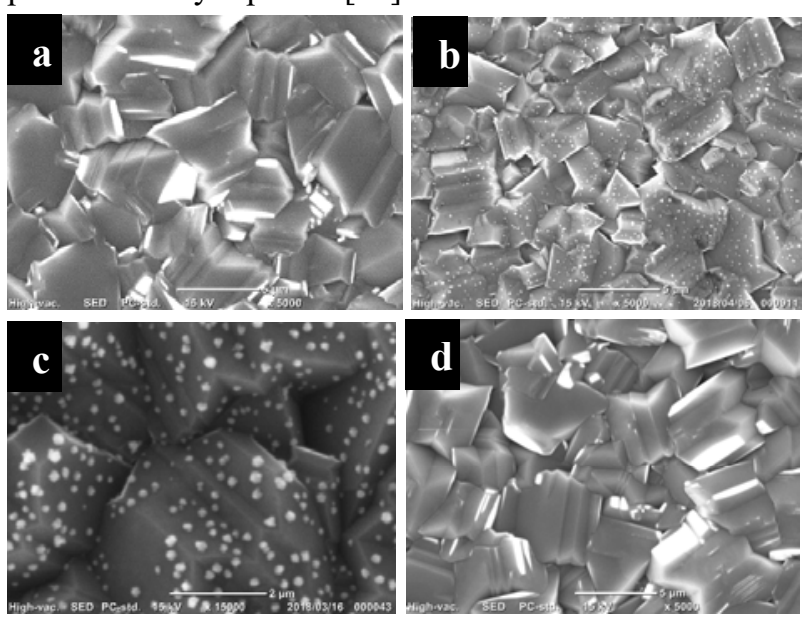

Fig. 1. SEM images of BDD (a), Cu-BDD (b: magnified 2000 times, c: magnified 5000 times) electrodes and recovered BDD (d) after modification with $\mathrm{B} / \mathrm{C}$ ratios of $1.0 \%$.

From EDX results, the percentage of $\mathrm{Cu}$ and $\mathrm{O}$ content were $1.99 \%$ and $0.33 \%$ for electrode after deposition (Table 1), while these two elements were not detected on the initial BDD surface, illustrating that copper was successfully deposited on BDD.

Table 1 Percentage of some elements for $\mathrm{Cu}$-BDD

\begin{tabular}{ccccc}
\hline Element & $\mathrm{C}$ & $\mathrm{B}$ & $\mathrm{O}$ & $\mathrm{Cu}$ \\
\hline $\begin{array}{c}\text { Percentage } \\
(\%)\end{array}$ & 95.79 & 1.89 & 0.33 & 1.99 \\
\hline
\end{tabular}

At the same time, Raman spectra were measured by Acton SP2500 (Princeton Instruments) with excitation at $532 \mathrm{~nm}$ from a green laser diode at room temperature before and after copper deposition, which was used to examine the chemical stability of BDD electrodes during electrodeposition and electrolysis. It could be found that Raman spectra for both unused and recovered BDD showed the peak of zone-center optical phonon of diamond at around $1300 \mathrm{~cm}^{-1}$ (Fig. 2). Two broad peaks were observed at around 500 and $1200 \mathrm{~cm}^{-1}$ without variation, which were typically observed in highly boron-doped diamond [14]. The result showed that BDD electrode was stable during electrodeposition and electrochemical nitrate reduction without any variation of chemical component.The results clearly illustrated that BDD electrode was benefit to particle application. 


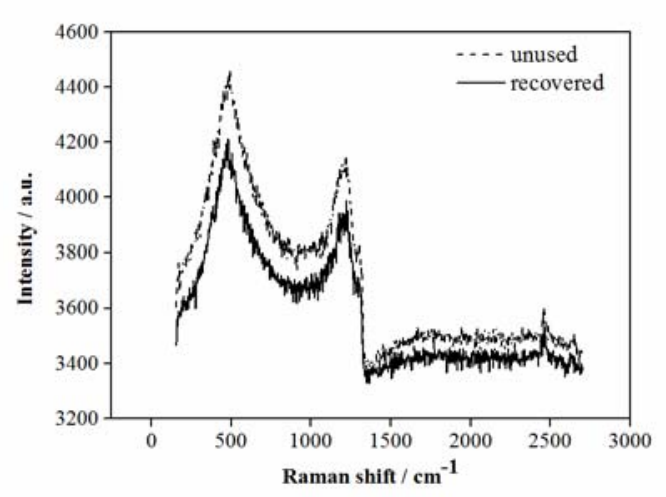

Fig. 2. Raman spectra of unused and recovered BDD electrode recorded at wavelength of $532 \mathrm{~nm}$.

\subsection{Behavior of electrochemical nitrate reduction}

Fig. 3 displays the linear sweep voltammograms (LSV) on $\mathrm{BDD}$ and $\mathrm{Cu}-\mathrm{BDD}$ in $0.1 \mathrm{M} \mathrm{Na}_{2} \mathrm{SO}_{4}$ aqueous solution with/without $10 \mathrm{mM} \mathrm{NaNO}$, at $100 \mathrm{mV} \mathrm{s}^{-1}$ were measured to compare the behavior of electrochemical nitrate reduction between BDD and $\mathrm{Cu}-\mathrm{BDD}$. Before each measurement, $\mathrm{Ar}$ gas was bubbled through the solution to remove dissolved oxygen, which could remove the dissolved oxygen to avoid oxygen reduction reaction (ORR) and sequential hinderance of nitrate reduction.

In Fig. 3b, no reduction peak was observed in the potentials ranging from 0 to $-2.0 \mathrm{~V}$ in $0.1 \mathrm{M} \mathrm{Na} 2 \mathrm{SO} 4$ solution without $\mathrm{NaNO}_{3}$ using $\mathrm{BDD}$, and the reduction peak at $-1.0 \mathrm{~V}$ for $\mathrm{Cu}-\mathrm{BDD}$ ascribed to the reduction of copper oxides on $\mathrm{Cu}$-BDD. Besides, HER occurred at around $-1.6 \mathrm{~V}$ for BDD electrode, while that for $\mathrm{Cu}$ electrode arose at more positive potential of $-1.25 \mathrm{~V}$. This result proved that $\mathrm{Cu}-\mathrm{BDD}$ also promoted HER owing to its higher conductivity.

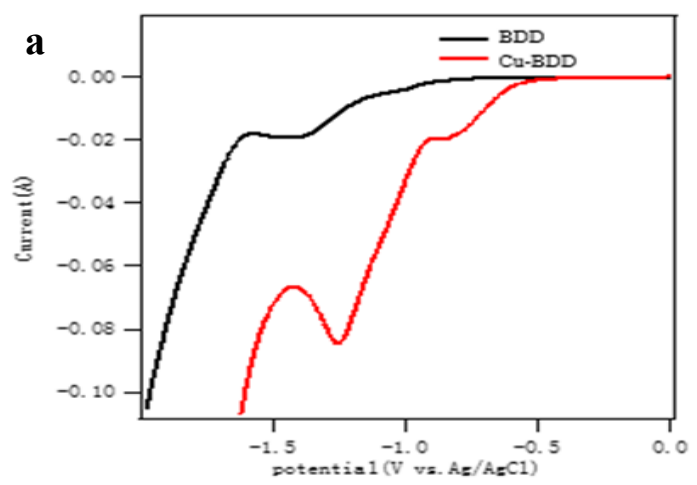

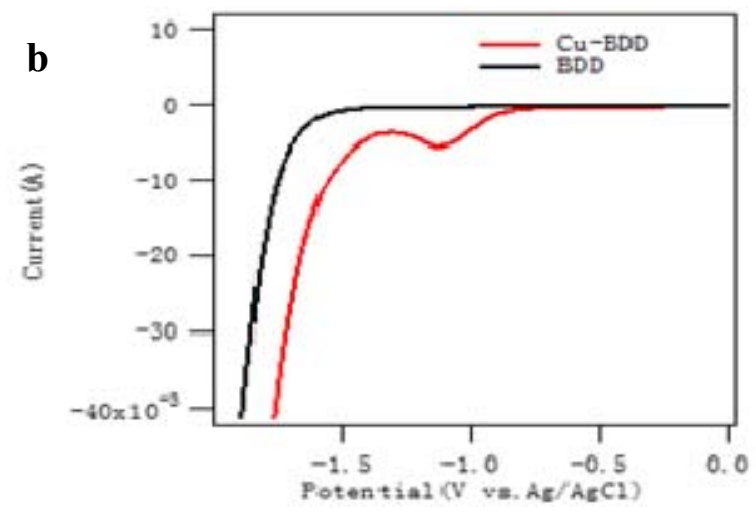

Fig. 3. $\mathrm{LSV}$ recorded at $100 \mathrm{mV} / \mathrm{s}$ with $\mathrm{BDD}$ (black) and $\mathrm{Cu}-$ $\mathrm{BDD}$ (red) in $0.1 \mathrm{M} \mathrm{Na}_{2} \mathrm{SO}_{4}$ solution with (a) and without (b) the presence of $10 \mathrm{mM} \mathrm{NaNO}_{3}$.

The clear reduction peaks could be seen at $-1.45 \mathrm{~V}$ for BDD and $-1.25 \mathrm{~V}$ for $\mathrm{Cu}-\mathrm{BDD}$ (Fig. 3a). Cu-BDD had the more positive potential than BDD for reducing nitrate, which was benefit to proceeding of the nitrate reduction. The results demonstrated that $\mathrm{Cu}-\mathrm{BDD}$ had the higher conductivity to promote electrochemical nitrate reduction. It might because the copper deposition decrease the potential window of BDD. Meanwhile, copper had the excellent catalytic ability also improved the electrochemical nitrate reduction [10]. On the other hand, the peak current of nitrate reduction for $\mathrm{Cu}-\mathrm{BDD}$ $(0.087 \mathrm{~A})$ was higher than that for BDD $(0.021 \mathrm{~A})$, which might be due to the higher activity of nitrate reduction with more electrons transfer using $\mathrm{Cu}-\mathrm{BDD}$. In addition, a reduction peak existed using $\mathrm{Cu}-\mathrm{BDD}$ at $-0.8 \mathrm{~V}$, which might attribute to the reduction of copper oxides, also proving that copper has been successfully deposited on BDD surface.

\subsection{Electrochemical nitrate reduction at different potentials}

Electrochemical nitrate reduction by BDD and Cu-BDD electrodes was studied at different potentials. The dependence of nitrate reduction efficiency and byproducts generation on the applied potential was obtained (Fig. 4). It could be found that Cu-BDD had the higher nitrate reduction efficiency than BDD at different potentials (Fig. 4a). For $\mathrm{Cu}-\mathrm{BDD}$, the nitrate reduction efficiency increased from $20 \%$ at $-1.2 \mathrm{~V}$ to the highest efficiency of $77 \%$ at $-2.0 \mathrm{~V}$, and then almost keep constant with the potential becoming more negative. While, the maximum efficiency was only $45 \%$ for BDD at $-2.0 \mathrm{~V}$. In terms of the generation of by-products, more ammonia produced using $\mathrm{Cu}-\mathrm{BDD}$ than that using $\mathrm{BDD}$, and large amount of nitrite generated during electrolysis for both $\mathrm{Cu}-\mathrm{BDD}$ and BDD (Fig. 4b).

The phenomenon could be explained that $\mathrm{Cu}-\mathrm{BDD}$ had the higher conductivity to improve the electron transfer and thus enhance the nitrate reduction efficiency. Meanwhile, the excellent catalytic ability of copper also could promote the electrochemical nitrate reduction process. Meanwhile, researchers have proved that the conversion from nitrate into nitrite was easier to occur, because of its two-electron reduction [11]. Therefore, the 
amount of nitrite was larger than that of ammonia. However, nitrite was unstable and easy to convert into some other nitrogenous substances [12], which resulted in the significant decrease in nitrite production. When the potential was more negative than $-2.0 \mathrm{~V}$, ammonia accumulation occurred using $\mathrm{Cu}-\mathrm{BDD}$. As our research found, $\mathrm{Cu}$-BDD promoted the occurrence of HER, which resulted in the high concentration of the adsorbed hydrogen (Hads) on the surface of $\mathrm{Cu}-\mathrm{BDD}$. Meanwhile, ammonia was proved to be a hydrogenated product of the nitrate reduction, and consequently hydrogen (Hads) on $\mathrm{Cu}-\mathrm{BDD}$ slightly increased ammonia accumulation. Many studies illustrated that adding $\mathrm{NaCl}$ could efficiently oxide ammonia into nitrogen gas or nitrate again, which is feasible for removing ammonia accumulation in our study.
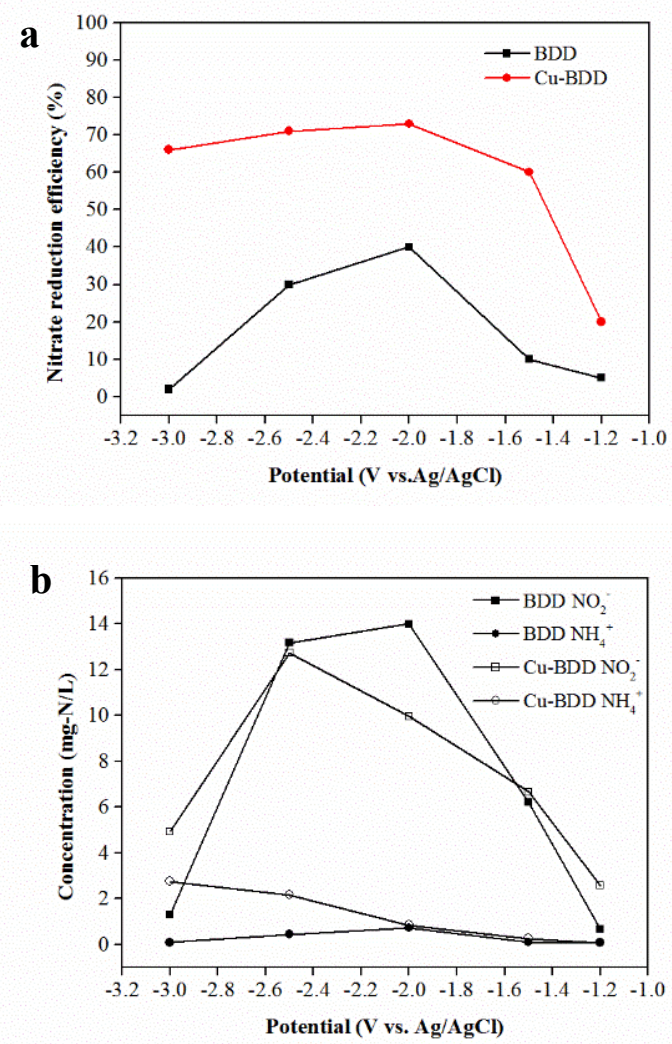

Fig. 4. Nitrate reduction efficiency (a) and the content of generated by-products (nitrite and ammonia) (b) for BDD and Cu-BDD.

\section{Conclusion}

In conclusion, the behavior of electrochemical nitrate reduction at $\mathrm{BDD}$ and $\mathrm{Cu}-\mathrm{BDD}$ electrodes was investigated by applying different potentials, and the results showed that the performance was strongly depended on the applied potential. The highest nitrate reduction efficiency about $77 \%$ was obtained at the optimal potential of $-2.0 \mathrm{~V}$ by using $\mathrm{Cu}-\mathrm{BDD}$. Results also showed that $\mathrm{Cu}-\mathrm{BDD}$ electrode enabled to reduce nitrate at more positive potential than BDD electrode. Copper modified BDD increased the conductivity of electrode to promote electrons transfer, and the excellent catalytic ability of copper was also in favour of nitrate reduction as well. This study shows that $\mathrm{Cu}$-BDD was more feasible for practical applications on the technology of electrochemical nitrate reduction owing to the higher conductivity.

\section{Acknowledgement}

The research was financed by the Science and Technology Innovation Foundation of Dalian, China (2018J12SN080) and Natural Science Foundation (National Innovation Joint Fund) of Liaoning, China. The Inorganic Materials Chemistry Laboratory, Keio University (KU). This research work was also supported by China Scholarship Council (CSC, No. 201706400005) during a visit of Peijing Kuang to KU, the Science and Technology Innovation Foundation of Dalian, China (2018J12SN080)

\section{References}

1. Y. Sun, M. Nemati, Bioresour. Technol., 114, 207 (2012)

2. M. Shrimali and K. P. Singh, Environ. Pollut., 112, 351 (2001)

3. S. K. Gupta, R. C. Gupta, A. B. Gupta, A. K. Seth, J. K. Bassin, A. Gupta, Environ. Health Persp, 108, 363 (2000).

4. D. Majumdar and N. Gupta, Indian J. Environ. Health, 42, 28 (2000).

5. P. Kuang, K. Natsui, Y. Einaga, Chemosphere 210, 524-530 (2018)

6. M. Christine, E. Michael, E. Craig, G. Richard, Anal. Sci. 21, 1430 (2005).

7. C. M. Welch, M. E. Hyde, C. E. Banks, R. G. Compton, Anal. Sci. 21, 1421(2005).

8. T. Yano, D. A. Tryk, K. Hashimoto A. Fujishima, J. Electrochem. Soc., 145, 1870-1876 (1998).

9. Natsui et al., 2018 K. Natsui, H. Iwakawa, N. Ikemiya, K. Nakata, Y. Einaga, Angew. Chem. Int. Ed. 57, 2639-2643 (2018).

10. K. Bouzek, M. Paidar, A. Sadílková, H. Bergmann, J. Appl. Electrochem. 31, 1185-1193 (2001).

11. C. Lévy-Clément, N. A. Ndao, A. Katty, M. Bernard, A. Deneuville, C. Comninellis, A. Fujishima, Diamond Relat. Mater. 12, 606-612 (2003).

12. C. Reuben, E. Galun, H. Cohen, R. Tenne, R. Kalish, Y. Muraki, K. Hashimoto, A. Fujishima, J.M. Butler, C. Lévy-Clément, J. Electroanal. Chem. 396, 233239 (1995).

13. K. Nakata, T. Ozaki, C. Terashima, A. Fujishima, Y. Einaga, Angew. Chem. Int. Ed. 53, 871-874 (2014).

14. F. Pruvost, E. Bustarret, A. Diamond Relat. Mater. 9, 295-299 (2000). 